\title{
Approche analytique de la limite de quantification pour le dosage du plomb sanguin : étude multicentrique
}

\section{Analytical approach of the limit of quantification of blood lead testing : a multicentric study}

\section{Didier OLICHON ${ }^{(1) * *}$, Laurence LABAT ${ }^{(2) *}$, Joël POUPON ${ }^{(3) *}$, Muriel BOST $^{(4) *}$, Vincent HAUFROID $^{(5) *}$, Christian MOESCH ${ }^{(6) *}$, Anne NICOLAS ${ }^{(7) \#}$, Yves FURET $^{(8)}$, Jean-Pierre GOULLÉ( ${ }^{(9)}$, Olivier GUILLARD ${ }^{(10)}$, Anne LE BOUIL ${ }^{(11)}$, Alain PINEAU ${ }^{(12)}$}

(1) Laboratoire Pasteur Cerba, Cergy Pontoise

(2) Laboratoire de Toxicologie et Génopathies, CHRU Lille (3) Laboratoire de Toxicologie Biologique, CHU Lariboisière, Paris (4) UF Pharmacologie et Analyse des Eléments Traces, CHU Lyon (5) Unité de Toxicologie Industrielle et Environnementale, Cliniques Universitaires Saint Luc, Bruxelles (Belgique)

(6) Hôpital Dupuytren, CHRU Limoges

(7) Laboratoire Toxilabo, Nantes

(8) Laboratoire de Pharmacologie, CHU Bretonneau, Tours (9) Laboratoire de Pharmacocinétique et de Toxicologie Clinique, GH du Havre

(10) Laboratoire de Biochimie et Toxicologie, CHU Poitiers

(11) Laboratoire de Pharmacologie et Toxicologie, CHU Angers

(12) Laboratoire de Toxicologie, Faculté de Pharmacie de Nantes

"Membres du groupe « Risques Toxicologiques » de la SFTA

*Auteur à qui adresser la correspondance : Didier OLICHON, Laboratoire Pasteur Cerba, 95066 Cergy Pontoise CEDEX 9

Tél : 0134402131 - Fax : 0134402019 - E-mail : dolichon@pasteur-cerba.com 


\section{RÉSUMÉ}

Les résultats du dosage du plomb sanguin pour le diagnostic et le suivi épidémiologique du saturnisme infantile en France sont exprimés différemment selon les laboratoires: il apparaît que la limite inférieure de rendu d'une valeur chiffrée correspond soit à la limite de détection (Ldd) soit à la limite de quantification $(\mathrm{Ldq})$ et que cette valeur est située dans une fourchette de 1 à $50 \mu \mathrm{g} / L$ selon le laboratoire et la méthode utilisée. A la demande de l'Institut de Veille Sanitaire (InVS), une étude multicentrique a été réalisée par le groupe de travail "Risques Toxicologiques » de la SFTA afin de proposer une méthode consensuelle de détermination de la Ldq du plomb sanguin et de répondre au problème de rendu des résultats pour les concentrations les plus faibles. Cette étude a été réalisée dans 12 laboratoires sur un pool de sangs prélevés sur EDTA et sélectionnés de manière à correspondre à une concentration peu élevée (de l'ordre de $40 \mu \mathrm{g} / \mathrm{L})$ pour préparer par dilution trois solutions sanguines de 20, 10 et $5 \mu \mathrm{g} / \mathrm{L}$. Ce pool et les trois solutions sanguines ont été analysés par l'ensemble des laboratoires par 12 méthodes de spectrométrie d'absorption atomique électrothermique $(S A A E)$ et par 4 méthodes de spectrométrie de masse en plasma induit (ICP-MS). L'objectif était d'approcher la Ldq par la concentration la plus faible quantifiée répondant à la limite d'acceptabilité de $20 \%$. Parallèlement, chaque laboratoire a déterminé sa Ldd et sa Ldq par 3 et 10 fois l'écart-type (ET) $)_{\text {blanc }}$ à partir du blanc (diluant) de sa méthode. Pour les 12 méthodes en SAAE, on observe des résultats comparables de la Ldq mesurée et de la Ldq calculée avec 6 méthodes. On peut noter une moindre variabilité dans la mesure de la Ldq par la méthode appliquant la limite des $20 \%$ (entre 4 et $18 \mu \mathrm{g} / \mathrm{L}$ ) que dans la méthode estimant la Ldq par $10 \mathrm{ET}_{\text {blanc }}$ (entre 1,6 et 27,7 $\mu \mathrm{g} / \mathrm{L}$ ). En ICP-MS, les coefficients de variation $C V$ restent toujours très inférieurs à $20 \%$ quelque soit le niveau de concentration mesurée et il n'est donc pas possible dans les conditions de cette étude de mesurer précisément la Ldq par la méthode de quantification avec la limite d'acceptabilité à 20\%. D'une façon générale, cette étude montre que l'approche de la Ldq par la méthode des $C V$ inférieurs à $20 \%$, bien que plus lourde à mettre en œuvre, semble plus réaliste. Elle permet également en SAAE de définir une Ldq et donc une limite de rendu des résultats proche d'une valeur de $15 \mu \mathrm{g} / \mathrm{L}$ pour trois méthodes et proche d'une valeur de $10 \mu \mathrm{g} / L$ pour 8 méthodes. En ICP-MS, les résultats montrent une meilleure sensibilité de la méthode pour le dosage du plomb sanguin avec une Ldq largement inférieure à $5 \mu \mathrm{g} / \mathrm{L}$ pour l'ensemble des laboratoires.

\section{MOTS-CLÉS}

Plomb, Sang, Limite de quantification, SAAE, ICP-MS.

\section{Introduction}

L'intoxication par le plomb, connue depuis des millénaires reste cependant toujours d'actualité. En raison des risques toxiques de ce métal (1), la surveillance nationale du saturnisme des enfants mineurs a été renforcée depuis 2004 (2) et comporte deux objectifs : une étude épidémiologique à l'échelon national s'appuyant sur les laboratoires d'analyses de biologie médicale et les

\section{SUMMARY}

In France, the results of blood lead concentration assays for the diagnosis and epidemiological monitoring of lead poisoning in children are expressed differently depending on the laboratory. It would appear that the significance level of the value issued corresponds either to the limit of detection (LOD) or the limit of quantification (LOQ) and that this value falls within a margin of 1 to $50 \mu \mathrm{g} / \mathrm{L}$ depending on the laboratory and the method used. A multicenter study was conducted by the SFTA's industrial toxic waste working group Risques Toxicologiques, at the behest of the Sanitary Surveillance Institute (Institut de Veille Sanitaire), in order to put forward a consensual method of calculating the LOQ of blood lead concentrations and to address the problem of issuing results for the weakest concentrations. The study was carried out in 12 laboratories on a blood pool drawn on EDTA and selected in order to correspond to an approximate concentration of $40 \mu \mathrm{g} / \mathrm{L}$ to prepare 3 blood solutions of 20, 10 and $5 \mu \mathrm{g} / \mathrm{L}$ per dilution. This pool and the 3 blood solutions were analysed by the laboratories using 12 electrothermal atomic absorption spectrometry (EAAS) methods and by 4 inductively coupled plasma mass spectrometry (ICP-MS) methods. The objective was to bring the LOQ closer to the weakest concentration quantified that met the $20 \%$ acceptability limit. In parallel, each laboratory identified its $L O D$ and its $L O Q$ with 3 and 10 times the standard deviation $(S D)_{\text {blank }}$ using a blank (diluent) from its method.

Of the 12 EAAS methods, similar results were observed between the measured LOQ and the calculated LOQ for 6 methods and it was observed that there were fewer differences for these 12 EAAS methods for $L O Q$ measurement by quantification with the $20 \%$ limit (between 4 and $18 \mu \mathrm{g} / \mathrm{L}$ ) than by calculation using the $10 S D_{\text {blank }}$ formula (between 1.7 and $27.7 \mu \mathrm{g} / \mathrm{L}$ ). In the case of ICP-MS, the CV always remained significantly below 20\% whatever the concentration level measured and it is therefore impossible in this study to estimate the $L O Q$ by the quantification method with a $20 \%$ acceptability limit. This study shows that the LOQ approach with a CV lower than $20 \%$ would seem to be more suitable. In the case of the EAAS method, it was possible to define a significance level for the result issued as being close to $15 \mu \mathrm{g} / \mathrm{L}$ (reported for 3 methods) and close to $10 \mu \mathrm{g} / \mathrm{L}$ (reported for 8 methods). In the case of ICP-MS the results reveal a better sensitivity for blood lead concentration assay methods with a $L O Q$ largely inferior to $5 \mu \mathrm{g} / \mathrm{L}$ for all the laboratories.

\section{KEY-WORDS}

Lead, Blood, EAAS, ICP-MS.

centres anti-poisons (CAP) et la déclaration obligatoire (DO) des cas de saturnismes s'appuyant sur les médecins prescripteurs et les DDASS. Ces objectifs sont atteints par la fiche unique de surveillance, de signalement et de notification (3), cette dernière étant effectuée pour un résultat de plombémie $>100 \mu \mathrm{g} / \mathrm{L}$.

Cependant il s'avère que l'introduction des résultats des plombémies des enfants dans la base de donnée anonyme des CAP de l'étude épidémiologique pilotée 
par l'Institut de Veille Sanitaire (InVS) pose le problème de l'homogénéité du rendu des résultats pour les valeurs basses. Il apparaît que la limite inférieure de rendu d'une valeur chiffrée correspond soit à la limite de détection (Ldd) soit à la limite de quantification (Ldq) et qu'elle est variable selon le laboratoire (de 1 à $50 \mu \mathrm{g} / \mathrm{L}$ ). A la demande de l'InVS, une étude multicentrique a été effectuée par le groupe de travail «Risques Toxicologiques » de la SFTA afin de proposer une méthode consensuelle de calcul de la Ldq du plomb sanguin et de répondre au problème de rendu des résultats pour les concentrations les plus faibles. Cette étude complète celle sur l'évaluation de l'incertitude de mesure de la plombémie pour les valeurs les plus basses (4).

Cette étude multicentrique a utilisé les méthodes actuellement en routine dans chacun des 12 laboratoires du groupe de travail, soit en spectrométrie d'absorption atomique électrothermique (SAAE), soit en spectrométrie de masse en plasma induit (ICP-MS). Elle a pour objectif d'approcher la Ldq par deux méthodes, une méthode de calcul par les écart-types du blanc et une autre méthode par la quantification de la concentration la plus faible respectant la limite d'acceptabilité de $20 \%$. La première, proposée par l'International Union of Pure and Applied Chemistry (IUPAC) en 1975 et 1995 (5), définit la limite de quantification comme étant égale à 10 fois l'écart-type des fluctuations du bruit de fond; elle est donc mesurée dans les conditions idéales et représente plutôt la limite de quantification instrumentale. La seconde méthode (Eurachem) basée sur la mesure de plusieurs solutions de concentrations décroissantes prend en compte l'ensemble de la méthode et est donc supposée mieux refléter la réalité. Ces deux approches ont été discutées dans le cadre du dosage du plomb sanguin en SAAE et ICP-MS (6).

\section{Matériel et méthodes}

\section{Les laboratoires participants}

L'étude a été réalisée dans 12 laboratoires (11 laboratoires français et 1 laboratoire belge) identifiés par des lettres de A à L. L'ensemble de ces laboratoires dose habituellement le plomb sanguin pour la surveillance du saturnisme infantile ou pour la surveillance de l'exposition professionnelle et participe à de nombreux contrôles de qualité (contrôle national obligatoire AFSSAPS dans le cadre de la surveillance professionnelle, programme de comparaisons inter laboratoires pour les métaux du Centre de Toxicologie du Québec, programme anglo-saxon TEQAS (Trace Elements External Quality Assessment Scheme) de «University of Surrey and Royal Surrey County Hospital,
Guilford, England »). Les plombémies ont été mesurées par SAAE (12 méthodes) dans 11 des laboratoires (ensemble des laboratoires sauf le laboratoire L) et en ICP-MS (4 méthodes) dans 4 des laboratoires (A, C, $H, L$ ). Le laboratoire $G$ a réalisé le dosage en double en SAAE sur deux appareils de générations différentes. Les résultats pour ce laboratoire seront codifiés par les lettres G1 et G2. Trois laboratoires (laboratoires A, C et $\mathrm{H})$ ont réalisé le dosage des plombémies par les deux méthodes : la SAAE et l'ICP-MS.

\section{Protocole de l'étude}

Le protocole de l'étude a été écrit et validé par l'ensemble des membres de la commission « Risques Toxicologiques » de la SFTA.

Un pool de sang prélevé sur EDTA a été constitué par un des laboratoires participant à l'étude. Le pool a été sélectionné de façon à obtenir une concentration ciblée située entre 40 et $50 \mu \mathrm{g} / \mathrm{L}$ pour éviter une trop grande modification de matrice lors des dilutions nécessaires à l'obtention de solutions sanguines filles faiblement titrées à 20, 10 et $5 \mu \mathrm{g} / \mathrm{L}$. Dans chacun des laboratoires le pool de départ et les 3 solutions sanguines titrées ont été analysés 20 fois en intra-série. Les moyennes et écart-types ont été mesurés pour chaque laboratoire. En parallèle, un test de répétabilité $(n=20)$ sur le blanc diluant de la méthode de dosage a été réalisé pour calculer la Ldd et la Ldq par la méthode des écart-types : $\mathrm{Ldd}=3 \mathrm{ET}_{\text {blanc }}$ et $\mathrm{Ldq}^{=} 10 \mathrm{ET}_{\text {blanc }}$.

\section{Méthodes de dosage}

Les appareils et accessoires utilisés ainsi que les principales caractéristiques des procédures opératoires de chaque laboratoire ont été préalablement décrits par Labat et coll. (4).

\section{Méthodes de calcul et d'estimation de la limite de quantification.}

La méthode de quantification du blanc (diluant mesuré 20 fois dans une même série) permet de calculer la limite de détection par la formule 3 ET du blanc $(\mathrm{Ldd}=3 \mathrm{ET})$ et la limite de quantification par la formule 10 ET du blanc (Ldq = 10 ET) (Tableau I).

La mesure répétée 20 fois du dosage du pool sanguin de départ et de chacune des solutions sanguines à 20, 10 et $5 \mu \mathrm{g} / \mathrm{L}$ permet une étude statistique avec moyenne, écart-type (ET) et coefficient de variation (CV) pour chaque niveau. L'extrapolation graphique de la courbe des $\mathrm{CV}$ obtenus en fonction des concentrations permet le calcul de la Ldq (figure 1). Dans chacun des tableaux correspondants aux mesures du pool de départ (tableau II) et des solutions filles sanguines (tableaux III, IV, V), les valeurs moyennes des plombémies sont déterminées pour chacun des laboratoires par la moyenne de 20 mesures pour chaque niveau de concentration. 


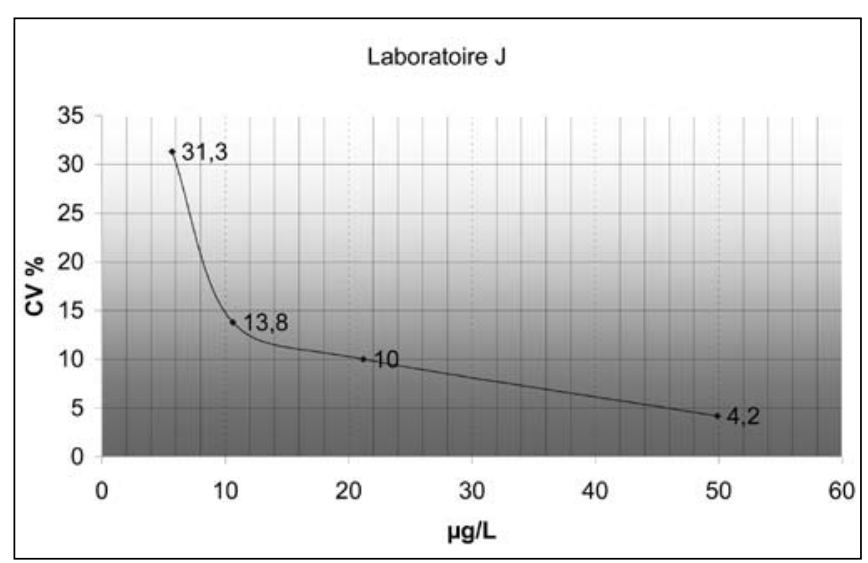

Figure 1 : Calcul de la Ldq pour le laboratoire J en SAAE, par l'étude de la relation $C V \%$ en fonction de la plombémie $(\mu \mathrm{g} / \mathrm{L})$.

Tableau I : Résultats du calcul de la $L d q(\mu g / L)$ par la méthode des blancs. (* résultats validés avec au minimum 2 décimales).

\begin{tabular}{|c|c|c|c|}
\hline $\begin{array}{c}\text { Laboratoires } \\
\text { SAAE }\end{array}$ & $\begin{array}{c}\text { Écart Type } \\
\text { du blanc }\end{array}$ & $\begin{array}{c}\text { Ldq } \\
(10 \text { ET }) \\
\end{array}$ & \\
\hline $\mathrm{A}$ & 0,166 & $1,66^{*}$ & \multirow{12}{*}{$\begin{array}{c}\text { Moyenne des } 12 \\
\text { méthodes en SAAE } \\
9,33 \mu \mathrm{g} / \mathrm{L} \\
(\mathrm{ET} 8,16)\end{array}$} \\
\hline B & 0,206 & $2,06^{*}$ & \\
\hline $\mathrm{C}$ & 1,845 & $18,45^{*}$ & \\
\hline D & 1,590 & $15,90 *$ & \\
\hline$E$ & 1,167 & 11,67 & \\
\hline $\mathrm{F}$ & 0,165 & $1,65^{*}$ & \\
\hline G1 & 0,791 & $7,91 *$ & \\
\hline G2 & 0,277 & $2,77 *$ & \\
\hline $\mathrm{H}$ & 2,769 & 27,69 & \\
\hline I & 0,86 & 8,60 & \\
\hline $\mathrm{J}$ & 1,1 & 11,00 & \\
\hline $\mathrm{K}$ & 0,264 & $2,64 *$ & \\
\hline $\begin{array}{c}\text { Laboratoires } \\
\text { ICP-MS }\end{array}$ & $\begin{array}{l}\text { Écart Type } \\
\text { du blanc }\end{array}$ & $\begin{array}{c}\text { Ldq } \\
(10 \text { ET })\end{array}$ & \\
\hline A & 0,003 & $0,03^{*}$ & \multirow{4}{*}{$\begin{array}{c}\text { Moyenne des } 4 \\
\text { méthodes en } \\
\text { ICP-MS } 0,83 \mu \mathrm{g} / \mathrm{L} \\
(\text { ET } 0,86)\end{array}$} \\
\hline C & 0,023 & $0,23^{*}$ & \\
\hline $\mathrm{H}$ & 0,122 & $1,22 *$ & \\
\hline $\mathrm{L}$ & 0,187 & $1,87^{*}$ & \\
\hline
\end{tabular}

Tableau II : Résultats obtenus par le pool sanguin initial.

\begin{tabular}{|c|c|c|c|c|}
\hline $\begin{array}{c}\text { Laboratoires } \\
\text { SAAE }\end{array}$ & $\begin{array}{l}\text { Moyenne } \\
\text { (ET) }\end{array}$ & CV\% & Exactitude & \\
\hline $\mathrm{A}$ & $54,72(5,05)$ & 9,23 & 109,69 & \multirow{12}{*}{$\begin{array}{c}\text { Moyenne des } \\
12 \text { méthodes } \\
\text { en SAAE } \\
50,01 \mu \mathrm{g} / \mathrm{L} \\
(\mathrm{CV} 13,16 \%\end{array}$} \\
\hline $\mathrm{B}$ & $47,87(1,51)$ & 3,15 & 95,56 & \\
\hline $\mathrm{C}$ & $47,37(2,85)$ & 6,01 & 94,96 & \\
\hline D & $47,41(2,57)$ & 5,41 & 95,04 & \\
\hline $\mathrm{E}$ & $52,93(0,98)$ & 1,85 & 106,11 & \\
\hline $\mathrm{F}$ & $44,00(1,08)$ & 2,44 & 88,20 & \\
\hline G1 & $56,99(2,85)$ & 5,00 & 114,24 & \\
\hline G2 & $46,96(4,61)$ & 9,82 & 94,13 & \\
\hline $\mathrm{H}$ & $46,23(3,08)$ & 6,66 & 92,68 & \\
\hline I & $46,05(1,19)$ & 2,58 & 92,32 & \\
\hline $\mathrm{J}$ & $44,61(1,89)$ & 4,24 & 89,42 & \\
\hline $\mathrm{K}$ & $64,94(2,99)$ & 4,61 & 130,18 & \\
\hline $\begin{array}{l}\text { Laboratoires } \\
\text { ICP-MS }\end{array}$ & $\begin{array}{l}\text { Moyenne } \\
\text { (ET) }\end{array}$ & CV\% & Exactitude & \\
\hline $\mathrm{A}$ & $51,34(2,22)$ & 4,33 & 102,92 & \multirow{4}{*}{$\begin{array}{c}\text { Moyenne des } \\
4 \text { méthodes } \\
\text { en ICP-MS } \\
49,52 \mu \mathrm{g} / \mathrm{L} \\
(\mathrm{CV} 6,76 \%)\end{array}$} \\
\hline $\mathrm{C}$ & $52,93(1,90)$ & 3,58 & 106,10 & \\
\hline $\mathrm{H}$ & $45,08(0,54)$ & 1,18 & 90,36 & \\
\hline $\mathrm{L}$ & $48,72(0,77)$ & 1,57 & 97,65 & \\
\hline
\end{tabular}

Tableau III : Résultats obtenus sur la solution sanguine $20 \mu \mathrm{g} / \mathrm{L}$.

\begin{tabular}{|c|c|c|c|c|}
\hline $\begin{array}{c}\text { Laboratoires } \\
\text { SAAE }\end{array}$ & $\begin{array}{l}\text { Moyenne } \\
\text { (ET) }\end{array}$ & CV\% & Exactitude & \\
\hline $\mathrm{A}$ & $23,44(2,49)$ & 10,63 & 110,44 & \multirow{12}{*}{$\begin{array}{c}\text { Moyenne des } \\
12 \text { méthodes } \\
\text { en SAAE } \\
21,34 \mu \mathrm{g} / \mathrm{L} \\
(\mathrm{CV} 12,94 \%)\end{array}$} \\
\hline $\mathrm{B}$ & $20,49(1,38)$ & 6,76 & 96,54 & \\
\hline $\mathrm{C}$ & $17,43(2,95)$ & 16,92 & 82,13 & \\
\hline $\mathrm{D}$ & $23,96(2,87)$ & 11,97 & 112,90 & \\
\hline $\mathrm{E}$ & $19,75(0,87)$ & 4,41 & 93,05 & \\
\hline $\mathrm{F}$ & $20,90(1,02)$ & 4,88 & 98,46 & \\
\hline G1 & $21,61(2,22)$ & 10,29 & 101,81 & \\
\hline G2 & $20,21(0,80)$ & 3,96 & 95,22 & \\
\hline $\mathrm{H}$ & $22,69(1,71)$ & 7,53 & 106,89 & \\
\hline $\mathrm{I}$ & $22,72(1,80)$ & 7,91 & 107,94 & \\
\hline $\mathrm{J}$ & $19,19(1,92)$ & 10,01 & 90,43 & \\
\hline $\mathrm{K}$ & $23,68(2,47)$ & 10,42 & 111,56 & \\
\hline $\begin{array}{c}\text { Laboratoires } \\
\text { ICP-MS }\end{array}$ & $\begin{array}{c}\text { Moyenne } \\
\text { (ET) }\end{array}$ & CV\% & Exactitude & \\
\hline $\mathrm{A}$ & $20,32(1,01)$ & 4,96 & 95,72 & \multirow{4}{*}{$\begin{array}{c}\text { Moyenne des } \\
4 \text { méthodes } \\
\text { en ICP-MS } \\
20,88 \mu \mathrm{g} / \mathrm{L} \\
(\mathrm{CV} 10,74 \%)\end{array}$} \\
\hline $\mathrm{C}$ & $21,56(0,94)$ & 4,37 & 101,57 & \\
\hline $\mathrm{H}$ & $18,06(0,27)$ & 1,51 & 85,09 & \\
\hline $\mathrm{L}$ & $23,59(1,43)$ & 6,07 & 111,13 & \\
\hline
\end{tabular}

Tableau IV : Résultats obtenus sur la solution sanguine $10 \mu \mathrm{g} / \mathrm{L}$.

\begin{tabular}{|c|c|c|c|c|c|}
\hline \multirow{2}{*}{\begin{tabular}{|c|} 
Laboratoires \\
SAAE
\end{tabular}} & \multirow{2}{*}{$\begin{array}{l}\text { Moyenne } \\
\text { (ET) }\end{array}$} & \multirow[t]{2}{*}{ CV\% } & \multicolumn{2}{|c|}{ Exactitude } & \\
\hline & & & Moyenne & Tronquée & \\
\hline $\mathrm{A}$ & $10,12(1,14)$ & $11,2 \%$ & $95,8 \%$ & $94,5 \%$ & \multirow{6}{*}{$\begin{array}{c}\text { Moyenne des } \\
12 \text { méthodes } \\
\text { en SAAE } \\
10,20 \mu \mathrm{g} / \mathrm{L} \\
(\mathrm{CV} 22,2 \%)\end{array}$} \\
\hline B & $9,79(0,65)$ & $6,7 \%$ & $92,7 \%$ & $91,4 \%$ & \\
\hline $\mathrm{C}$ & $7,11(2,57)$ & $36,2 \%$ & $67,3 \%$ & $66,4 \%$ & \\
\hline $\mathrm{D}$ & $11,68(2,58)$ & $22,1 \%$ & $110,5 \%$ & $109,0 \%$ & \\
\hline E & $9,96(0,75)$ & $7,5 \%$ & $94,3 \%$ & $93,0 \%$ & \\
\hline $\mathrm{F}$ & $11,07(0,96)$ & $8,7 \%$ & $104,8 \%$ & $103,3 \%$ & \\
\hline G1 & $10,92(2,03)$ & $18,6 \%$ & $103,4 \%$ & $101,9 \%$ & \multirow{6}{*}{$\begin{array}{c}\text { Moyenne des } \\
12 \text { méthodes } \\
\text { en SAAE } \\
10,25 \mu \mathrm{g} / \mathrm{L} \\
(\mathrm{CV} 16,7 \%)\end{array}$} \\
\hline G2 & $8,77(0,52)$ & $5,9 \%$ & $83,1 \%$ & $81,9 \%$ & \\
\hline $\mathrm{H}$ & $10,03(2,36)$ & $23,6 \%$ & $94,9 \%$ & $96,6 \%$ & \\
\hline I & $11,64(2,57)$ & $22,1 \%$ & $110,2 \%$ & $108,7 \%$ & \\
\hline $\mathrm{J}$ & $12,47(1,73)$ & $13,8 \%$ & $118,1 \%$ & $116,4 \%$ & \\
\hline $\mathrm{K}$ & $8,87(1,67)$ & $18,9 \%$ & $84,0 \%$ & $82,8 \%$ & \\
\hline \multirow{2}{*}{$\begin{array}{c}\text { Laboratoires } \\
\text { ICP-MS }\end{array}$} & \multirow{2}{*}{$\begin{array}{l}\text { Moyenne } \\
\text { (ET) }\end{array}$} & \multirow{2}{*}{ CV\% } & \multicolumn{2}{|c|}{ Exactitude } & \\
\hline & & & Moyenne & Tronquée & \\
\hline A & $12,01(0,33)$ & $2,8 \%$ & $113,7 \%$ & $112,1 \%$ & \multirow{4}{*}{$\begin{array}{l}\text { Moyenne des } \\
4 \text { méthodes } \\
\text { en ICP-MS } \\
11,64 \mu \mathrm{g} / \mathrm{L} \\
(\mathrm{CV} 7,6 \%)\end{array}$} \\
\hline $\mathrm{C}$ & $12,37(0,7)$ & $5,6 \%$ & $117,1 \%$ & $115,5 \%$ & \\
\hline $\mathrm{H}$ & $10,32(0,21)$ & $2,0 \%$ & $97,7 \%$ & $96,4 \%$ & \\
\hline $\mathrm{L}$ & $11,87(0,26)$ & $2,2 \%$ & $112,4 \%$ & $110,8 \%$ & \\
\hline
\end{tabular}

Les exactitudes sont calculées pour chaque méthode rapportée à la valeur moyenne cible correspondant à l'ensemble des mesures effectuées, toutes méthodes confondues, pour chaque niveau.

\section{Résultats et Discussion}

Le tableau I résume les résultats obtenus pour le calcul de l'ET et le calcul de la Ldq à partir du blanc de la méthode. Pour les 12 méthodes utilisant la SAAE, la moyenne des Ldq calculées est de 9,33 $\mu \mathrm{g} / \mathrm{L}$ et pour les 4 méthodes utilisant l'ICP-MS, la moyenne est de $0,83 \mu \mathrm{g} / \mathrm{L}$. On note cependant une très grande dispersion 
Tableau V: Résultats obtenus sur la solution sanguine $5 \mu \mathrm{g} / \mathrm{L}$.

\begin{tabular}{|c|c|c|c|c|c|}
\hline \multirow{2}{*}{\begin{tabular}{|c} 
Laboratoires \\
SAAE
\end{tabular}} & \multirow{2}{*}{$\begin{array}{l}\text { Moyenne } \\
\text { (ET) }\end{array}$} & \multirow{2}{*}{ CV\% } & \multicolumn{2}{|c|}{ Exactitude } & \\
\hline & & & Moyenne & Tronquée & \\
\hline $\mathrm{A}$ & $7,32(1,49)$ & $20,3 \%$ & $129,4 \%$ & $112,5 \%$ & \multirow{6}{*}{$\begin{array}{c}\text { Moyenne des } \\
12 \text { méthodes } \\
\text { en SAAE } \\
5,32 \mu \mathrm{g} / \mathrm{L} \\
(\mathrm{CV} 44,1 \%)\end{array}$} \\
\hline B & $3,68(1,48)$ & $40,3 \%$ & $65,1 \%$ & $56,6 \%$ & \\
\hline $\mathrm{C}$ & $2,49(1,86)$ & $74,7 \%$ & $44,0 \%$ & $38,3 \%$ & \\
\hline $\mathrm{D}$ & $5,5(1,46)$ & $26,6 \%$ & $97,1 \%$ & $84,4 \%$ & \\
\hline $\mathrm{E}$ & $5,88(0,91)$ & $15,5 \%$ & $103,8 \%$ & $90,3 \%$ & \\
\hline $\mathrm{F}$ & $7,18(1,31)$ & $18,3 \%$ & $126,9 \%$ & $110,3 \%$ & \\
\hline G1 & $6,16(2,37)$ & $38,4 \%$ & $108,9 \%$ & $94,7 \%$ & \multirow{6}{*}{$\begin{array}{c}\text { Moyenne des } \\
12 \text { méthodes } \\
\text { en SAAE } \\
6,29 \mu \mathrm{g} / \mathrm{L} \\
(\mathrm{CV} 19,0 \%)\end{array}$} \\
\hline G2 & $5,81(0,8)$ & $13,9 \%$ & $102,6 \%$ & $89,2 \%$ & \\
\hline $\mathrm{H}$ & $6,36(1,64)$ & $25,8 \%$ & $112,3 \%$ & $97,6 \%$ & \\
\hline $\mathrm{I}$ & $1,7(1,95)$ & $114,6 \%$ & $30,0 \%$ & $26,1 \%$ & \\
\hline $\mathrm{J}$ & $6,66(2,08)$ & $31,3 \%$ & $117,6 \%$ & $102,2 \%$ & \\
\hline K & $5,14(1,55)$ & $30,3 \%$ & $90,7 \%$ & $78,9 \%$ & \\
\hline \multirow{2}{*}{$\begin{array}{c}\text { Laboratoires } \\
\text { ICP-MS }\end{array}$} & \multirow{2}{*}{$\begin{array}{l}\text { Moyenne } \\
\text { (ET) }\end{array}$} & \multirow{2}{*}{ CV\% } & \multicolumn{2}{|c|}{ Exactitude } & \\
\hline & & & Moyenne & Tronquée & \\
\hline A & $5,77(0,09)$ & $1,5 \%$ & $101,9 \%$ & $88,6 \%$ & \multirow{4}{*}{$\begin{array}{c}\text { Moyenne des } \\
4 \text { méthodes } \\
\text { en ICP-MS } \\
6,67 \mu \mathrm{g} / \mathrm{L} \\
\text { (CV } 10,0 \%)\end{array}$} \\
\hline $\mathrm{C}$ & $7,26(0,32)$ & $4,4 \%$ & $128,2 \%$ & $111,5 \%$ & \\
\hline $\mathrm{H}$ & $6,78(0,62)$ & $9,2 \%$ & $119,8 \%$ & $104,2 \%$ & \\
\hline $\mathrm{L}$ & $6,89(0,26)$ & $3,8 \%$ & $121,8 \%$ & $105,9 \%$ & \\
\hline
\end{tabular}

Tableau VI : Récapitulatif des CV \% selon les niveaux de concentration (moyenne calculée sur tous les résultats obtenus en SAAE et ICP-MS).

\begin{tabular}{|c|c|c|c|c|}
\hline & $\begin{array}{c}\text { Pool initial } \\
\text { (moyenne 49,9) }\end{array}$ & $\begin{array}{c}\text { Cible } 20 \mu \mathrm{g} / \mathrm{L} \\
\text { (moyenne 21,2) }\end{array}$ & \begin{tabular}{|c|} 
Cible $10 \mu \mathrm{g} / \mathrm{L}$ \\
(moyenne 10,6)
\end{tabular} & $\begin{array}{c}\text { Cible } 5 \mu \mathrm{g} / \mathrm{L} \\
\text { (moyenne 5,7) }\end{array}$ \\
\hline $\mathrm{A}$ & $9,2 \%$ & $10,6 \%$ & $11,2 \%$ & $20,3 \%$ \\
\hline $\mathrm{B}$ & $3,2 \%$ & $6,8 \%$ & $6,7 \%$ & $40,3 \%$ \\
\hline $\mathrm{C}$ & $6,0 \%$ & $16,9 \%$ & $36,2 \%$ & $74,7 \%$ \\
\hline $\mathrm{D}$ & $5,4 \%$ & $12,0 \%$ & $22,1 \%$ & $26,6 \%$ \\
\hline $\mathrm{E}$ & $1,9 \%$ & $4,4 \%$ & $7,5 \%$ & $15,5 \%$ \\
\hline $\mathrm{F}$ & $2,4 \%$ & $4,9 \%$ & $8,7 \%$ & $18,3 \%$ \\
\hline G1 & $5,0 \%$ & $10,3 \%$ & $18,6 \%$ & $38,4 \%$ \\
\hline $\mathrm{G} 2$ & $9,8 \%$ & $4,0 \%$ & $5,9 \%$ & $13,9 \%$ \\
\hline $\mathrm{H}$ & $6,7 \%$ & $7,5 \%$ & $23,6 \%$ & $25,8 \%$ \\
\hline $\mathrm{I}$ & $2,6 \%$ & $7,9 \%$ & $22,1 \%$ & $114,6 \%$ \\
\hline $\mathrm{J}$ & $4,2 \%$ & $10,0 \%$ & $13,8 \%$ & $31,3 \%$ \\
\hline $\mathrm{K}$ & $4,6 \%$ & $10,4 \%$ & $18,9 \%$ & $30,3 \%$ \\
\hline $\mathrm{A}-\mathrm{ICP}$ & $4,3 \%$ & $5,0 \%$ & $2,8 \%$ & $1,5 \%$ \\
\hline C - ICP & $3,6 \%$ & $4,4 \%$ & $5,6 \%$ & $4,4 \%$ \\
\hline $\mathrm{H}-\mathrm{ICP}$ & $1,2 \%$ & $1,5 \%$ & $2,0 \%$ & $9,2 \%$ \\
\hline $\mathrm{L}-\mathrm{ICP}$ & $1,6 \%$ & $6,1 \%$ & $2,2 \%$ & $3,8 \%$ \\
\hline
\end{tabular}

des Ldq avec les méthodes en SAAE variant de 1,65 à $27,69 \mu \mathrm{g} / \mathrm{L}$. En revanche, les résultats obtenus avec les méthodes en ICP-MS confirment la plus grande sensibilité de ces méthodes avec une moyenne des Ldq plus faible qu'en SAAE.

Le tableau II regroupe pour chaque laboratoire les résultats statistiques (moyenne, $\mathrm{ET}$ et $\mathrm{CV} \%$ ) obtenus pour les 20 mesures du pool de sang de départ. La moyenne des 240 mesures de l'ensemble des laboratoires ayant utilisé une méthode en SAAE est de 50,01 $\mu \mathrm{g} / \mathrm{L}$ et la moyenne des 80 mesures de l'ensemble des laboratoires ayant utilisé une méthode en ICP-MS est de 49,52 $\mu \mathrm{g} / \mathrm{L}$, démontrant une excellente corrélation à ce niveau de concentration entre les 2 méthodes. L'exactitude de chaque laboratoire par rapport à la valeur cible (moyenne des 240 mesures en SAAE et moyenne des 80 mesures en ICP-MS) est exprimée en \%.

Les tableaux III, IV et $\mathrm{V}$ regroupent les mêmes éléments statistiques pour les solutions sanguines ciblées à 20, 10 et $5 \mu \mathrm{g} / \mathrm{L}$. Pour la concentration théorique de $20 \mu \mathrm{g} / \mathrm{L}$, la moyenne des 240 mesures de l'ensemble des laboratoires ayant utilisé une méthode en SAAE est de 21,34 $\mu \mathrm{g} / \mathrm{L}$ tandis que la moyenne des 80 mesures de l'ensemble des laboratoires ayant utilisé une méthode en ICP-MS est de 20,88 $\mu \mathrm{g} / \mathrm{L}$. On notera que pour cette concentration, tous les laboratoires ont un CV $<20 \%$. En revanche pour la concentration théorique de $10 \mu \mathrm{g} /$ $\mathrm{L}$, seulement 8 méthodes en SAAE sur les 12 obtiennent un $\mathrm{CV}<20 \%$, et pour la valeur cible de $5 \mu \mathrm{g} / \mathrm{L}$ il n'y a plus que 3 méthodes en SAAE qui satisfont à la limite d'acceptabilité de $20 \%$.

Tableau VII : Comparaison des modes de calcul de la Ldq pour les méthodes en SAAE.

\begin{tabular}{|c|c|c|c|c|c|}
\hline Laboratoire & $\begin{array}{c}\text { Mesure (R1) de } \\
\text { la Ldq par la } \\
\text { méthode à 20 \% }\end{array}$ & $\begin{array}{c}\text { Calcul (C2)de } \\
\text { la Ldq par la } \\
\text { formule Ldq } \\
\text { = 10 ET }\end{array}$ & \multicolumn{4}{|c|}{$\begin{array}{c}\text { Rapport des Ldq } \\
\text { R1/C2 }\end{array}$} \\
\hline A & 6,5 & 1,66 & 3,9 & & \\
\hline B & 8 & 2,06 & 3,9 & & \\
\hline C & 18 & 18,45 & & 1,0 & \\
\hline D & 12,5 & 15,90 & & 0,8 & \\
\hline E & 5 & 11,67 & & & 0,4 \\
\hline F & 6 & 1,65 & 3,6 & & \\
\hline G1 & 10 & 7,91 & & 1,3 & \\
\hline G2 & 4 & 2,77 & & 1,4 & \\
\hline H & 12,5 & 27,69 & & & 0,5 \\
\hline I & 11 & 8,60 & & 1,3 & \\
\hline J & 8 & 11,00 & & 0,7 & \\
\hline K & 10 & 2,64 & 3,8 & & \\
\hline
\end{tabular}

Seules les 80 mesures de l'ensemble des laboratoires ayant utilisé une méthode en ICP-MS pour la valeur théorique de $5 \mu \mathrm{g} / \mathrm{L}$ permettent d'obtenir pour une moyenne de 6,67 $\mu \mathrm{g} / \mathrm{L}$, un CV de 10,0\% (tableau V). A ce niveau de concentration le plus faible de notre étude, toutes les méthodes en ICP-MS satisfont à la limite d'acceptabilité.

Pour chacun des laboratoires, le tableau VI résume les performances obtenues pour les différents niveaux de concentrations de l'étude, et le tableau VII résume les deux méthodes de détermination de la Ldq.

Enfin, dans l'exemple des résultats en SAAE du laboratoire J, la figure 1 montre le mode de calcul de la Ldq à l'aide de la relation CV \% / concentration $\mu \mathrm{g} / \mathrm{L}$. Ce mode de détermination graphique n'a pas pu être utilisé avec les méthodes en ICP-MS et raison des CV toujours inférieurs à $20 \%$ (figure 2). 


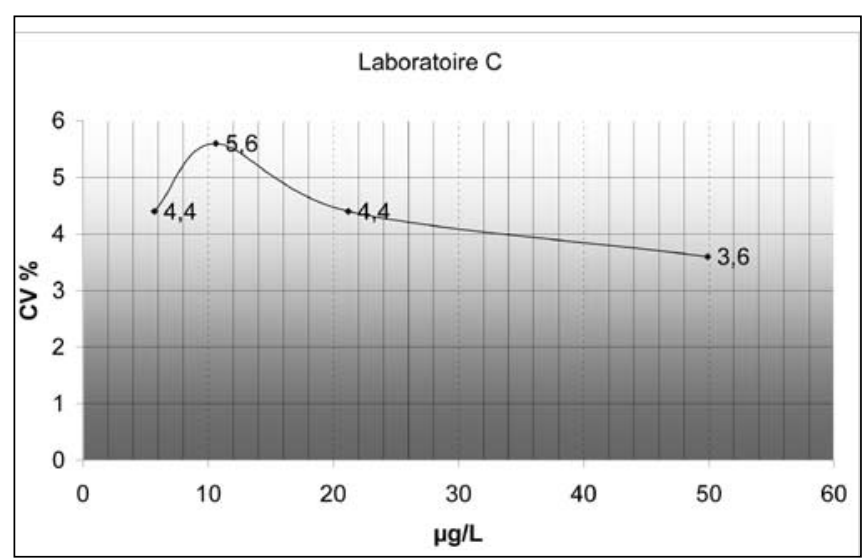

Figure 2 : Calcul de la Ldq pour le laboratoire C en ICP-MS, par l'étude de la relation $C V \%$ en fonction de la plombémie $(\mu \mathrm{g} / L)$. (non mesurable par cette méthode).

En SAAE, les deux modes de calcul de la Ldq donnent des résultats comparables pour six laboratoires (C, D, G1, G2, I et J). On observe moins de différence de mesure de la Ldq entre les 12 laboratoires par la méthode des CV (entre 4 et $18 \mu \mathrm{g} / \mathrm{L}$ ) que par la méthode de calcul utilisant les blancs (entre 1,7 et 27,7 $\mu \mathrm{g} / \mathrm{L}$ ). De plus, les résultats sont souvent plus élevés avec cette seconde méthode de calcul ce qui était prévisible car la première ne représente que la Ldq instrumentale tandis que la seconde inclue l'ensemble de la méthode. En ICP-MS, les CV restent toujours très inférieurs à $20 \%$ et il n'est donc pas possible dans cette étude d'estimer précisément la Ldq par la méthode de calcul des CV. Les résultats de cette étude suggèrent que les Ldq en ICP-MS sont situées très au dessous de $5 \mu \mathrm{g} / \mathrm{L}$.

\section{Conclusion}

D’une façon générale, la détermination de la Ldq du dosage de la plombémie dans les laboratoires ayant participé à cette étude apparaît plus réaliste, bien que plus fastidieuse, par la mesure quantitative avec la méthode des $\mathrm{CV}$ inférieurs à $20 \%$. Elle permet en SAAE de définir une limite de rendu des résultats proche d'une valeur de $15 \mu \mathrm{g} / \mathrm{L}$ (observée pour 11 méthodes), limite que l'on peut espérer améliorer dans l'avenir vers une valeur à $10 \mu \mathrm{g} / \mathrm{L}$ (actuellement observée pour 8 méthodes). Ces premiers résultats en ICP-MS montrent une meilleure sensibilité de la méthode pour le dosage du plomb sanguin avec une Ldq largement inférieure à $5 \mu \mathrm{g} / \mathrm{L}$ pour l'ensemble des laboratoires utilisant cette technologie. Une nouvelle approche apparaît indispensable et permettrait de mieux apprécier la Ldq de cette dernière technique.

\section{Références}

1. Garnier R. Toxicité du plomb et des ses dérivés. EMC - Toxicologie-Pathologie professionnelle 2005 ; 16-007A-10, 15 p.

2. Arrêté du 5 février 2004 relatif à la déclaration obligatoire du saturnisme de l'enfant mineur. J.O. 5 mars 2004 ; pp. 4390-4391.

3. Surveillance des plombémies. Saturnisme chez l'enfant mineur.http://www.invs.sante.fr/surveillance/saturnisme/ fiche_do_saturnisme.pdf

4. Labat L., Olichon D., Poupon J., Bost M., Haufroid V., Moesch C., Nicolas A., Furet Y., Goullé J.-P., Guillard O., Le Bouil A., Pineau A. Variabilité de la mesure de la plombémie pour de faibles concentrations proches du seuil de $100 \mu \mathrm{g} / \mathrm{L}$ : étude multicentrique. Ann. Toxicol. Anal., 2006, 18 : 297-304.

5. Currie LA. Nomenclature in evaluation of analytical methods including detection and quantification capabilities (IUPAC recommendations 1995). Pure Appl Chem 1995 ; 67 : 1699-723.

6. Bonnefoy C., Menudier A., Moesch C., Lachâtre G., Mermet J.-M. Validation of the determination of lead in whole blood by ICP-MS. J. Anal. At. Spectrom., 2002, $17: 1161-1165$. 